\title{
MicroRNA-200 family members and ZEB2 are associated with brain metastasis in gastric adenocarcinoma
}

\author{
YANG-KI MINN ${ }^{1}$, DA HYE LEE ${ }^{2}$, WOO JIN HYUNG ${ }^{3}$, JI EUN KIM ${ }^{4}$, JUNJEONG CHOI ${ }^{5}$, \\ SEUNG-HO YANG ${ }^{6}$, HOTAEK SONG ${ }^{7}$, BEOM JIN LIM ${ }^{2}$ and SE HOON KIM ${ }^{2}$ \\ ${ }^{1}$ Department of Neurology, Kangnam Sacred Heart Hospital, Hallym University, Seoul; \\ Departments of ${ }^{2}$ Pathology and ${ }^{3}$ Surgery, Yonsei University, College of Medicine, Seoul; ${ }^{4}$ Department of Pathology, \\ Seoul National University, Boramae Hospital, Seoul; ${ }^{5}$ Department of Pathology, Yonsei University, Wonju College \\ of Medicine, Wonju; ${ }^{6}$ Department of Neurosurgery, St. Vincent Hospital, The Catholic University of Korea, Suwon; \\ ${ }^{7}$ Department of Radiology and Research Institute of Radiologic Science, Yonsei University, \\ College of Medicine, Seoul, Republic of Korea
}

Received August 2, 2014; Accepted September 16, 2014

DOI: $10.3892 / \mathrm{ijo} .2014 .2680$

\begin{abstract}
Although the incidence of brain metastasis in gastric cancer is relatively low, its prevalence may increase with improved therapy and longer survival tumors. The molecular mechanisms underlying brain metastases are not well understood. To gain insight into the mechanism of brain metastasis, we studied differences in microRNA (miRNA) expression levels in 8 cases of matched primary gastric adenocarcinoma and brain metastatic adenocarcinoma using the Illumina microRNA microarray chip. We identified 6 upregulated and 2 downregulated miRNAs in all 8 cases simultaneously. Interestingly, 2 out of 8 miRNAs (hsa-miR-141-3p and hsa-miR-200b-3p) belonged to the miR-200 family. Online microRNA database searching revealed that ZEB2 is the topranked target gene for hsa-miR141-3p and hsa-miR-200b-3p, prompting us to focus ZEB2 expression in brain metastatic adenocarcinoma. We confirmed that ZEB2 expression was markedly downregulated in some brain metastatic samples. In addition, decreased ZEB2 expression was noted by western blot analysis of 2 metastatic gastric adenocarcinoma cell types that were derived by in vivo selection following intracardiac injection of gastric cancer cell lines. In conclusion, we demonstrate that expression of miRNA-200 family members and ZEB2 are associated with brain metastases of gastric adenocarcinoma, not only in matched patient samples, but also in metastatic cell lines that were derived by in vivo selection.
\end{abstract}

Correspondence to: Professor Se Hoon Kim, Department of Pathology, Yonsei University College of Medicine 50-1 Yonsei-ro, Seodaemun-gu, Seoul 120-752, Republic of Korea E-mail: paxco@yuhs.ac

Key words: metastasis, gastric, adenocarcinoma, microRNA

\section{Introduction}

Although the incidence of gastric cancer has been declining since 1950, gastric cancer remains the 4 th most common cancer worldwide (1). The prevalence of gastric cancer shows wide geographic variations, with the highest rates found in Eastern Asia (including China, Japan and Korea), Eastern Europe, and Central and Latin America (1). In a recent report by the World Health Organization, gastric cancer was classified into 5 main types of adenocarcinomas and rare variants (2). In contrast to the better prognoses associated with early gastric cancers, advanced gastric cancers generally have poor prognoses, with known prognostic factors including stage and the number of lymph node metastases (2). Unlike lung or breast cancer, the brain metastases of gastric cancer are very rare and have been reported in $<1 \%$ of clinical cases $(3,4)$, most cases of which were reported as leptomeningeal carcinomatosis (5-7).

Prolonged survival in gastric cancer patients accompanying improvements in systematic approaches and overall patient care has increased the likelihood of patients developing central nervous system metastases (8). Similarly, the incidence of brain metastasis in non-small cell lung cancer patients has increased in recent years (9), likely resulting from improved therapy, diagnostic modalities, and screening programs for early detection. In addition, evidence suggests that improved targeted therapy in patients with HER-2-positive breast cancer may increase the incidence of brain metastases $(10,11)$. Although there is no established targeted therapy for treating gastric cancer, novel therapeutics are being actively investigated in clinical trials $(12,13)$. Due to therapeutic progress and the emergence of targeted therapy, a reasonable concern is that the incidence of brain metastasis in gastric cancer may rise in response. However, the molecular mechanism of brain metastases is currently not well understood.

To study the mechanism of brain metastases development, it is essential to identify the molecular differences between primary and metastatic lesions. In contrast of breast or lung carcinoma, most gastric cancer brain metastases display a 
tendency for leptomeningeal spread, and surgical treatment options for brain lesions may be limited. Thus, opportunities for acquiring brain metastatic gastric cancer tissues, especially fresh tissues are very limited.

MicroRNA (miRNAs) comprise of a broad class of small, non-coding RNAs that negatively regulate the expression of hundreds of target genes, thereby controlling a wide range of biological functions, including those performed by oncogenes or tumor suppressor genes (14). In addition, some evidence has indicated that miRNAs may have key roles in regulating tumor cell invasion and metastases (15).

Formalin-fixed, paraffin-embedded (FFPE) tissue samples are an invaluable resource for the study of human diseases (16). In FFPE tissues, RNA is fragmented and may be chemically modified, making it unsuitable for research. However, with advances of technologies, miRNA expression profiles from FFPE tissues closely resemble those from fresh tissues (17). In the present study, we investigated differences of miRNA profiles between matched brain metastatic gastric adenocarcinoma and primary gastric cancer to gain insight into the mechanisms of brain metastases.

\section{Materials and methods}

Tissue samples. We reviewed pathology reports of 3 hospitals (Severance Hospital, Gang Nam Severance Hospital and Seoul National University Hospital) recorded between 2000 and 2012 and found archival FFPE blocks from 8 cases of primary gastric adenocarcinoma with corresponding brain metastases. After 3 pathologists involved in the present study (S.H. Kim, J.E. Kim and B.J. Lim) reviewed the cases, we selected representative tissue blocks from 8 gastric adenocarcinoma cases and 8 corresponding brain metastatic cases. This study was approved by the Institutional Review Board of Medicine (4-2012-0346) of Severance Hospital.

Tissue preparations. Tumor areas on paraffin blocks were marked and microdissected to allow enrichment of tumor fractions by $>70 \%$. For each specimen, 3 microdissected tissue sections (10- $\mu \mathrm{m}$ thickness) were obtained and collected for RNA extraction. Total RNA was isolated using the mirVana ${ }^{\mathrm{TM}}$ miRNA Isolation kit (Ambion, Austin, TX, USA) according to the manufacturer's instructions. Extracted RNA was quantitated using a NanoDrop 1000 Spectrophotometer (Thermo Fisher Scientific, Inc., Wilmington, DE, USA).

Agilent miRNA microarrays methods. Microarray studies were performed using the miRNA Microarray System with the miRNA Complete Labeling and Hybridization kit (Agilent Technologies, Santa Clara, CA, USA), according to the manufacturer's recommended protocol. The Agilent microRNA Spike-In kit was used as a sample process control to measure labeling and hybridization efficiency.

Briefly, $100 \mathrm{ng}$ total RNA was dephosphorylated at $37^{\circ} \mathrm{C}$ for $30 \mathrm{~min}$ with calf intestinal phosphatase (Invitrogen/Life Technologies, Carlsbad, CA, USA) and denatured in $100 \%$ dimethyl sulfoxide at $100^{\circ} \mathrm{C}$ for $7 \mathrm{~min}$. Samples were labeled with pCp-Cy3 and T4 ligase (Invitrogen/Life Technologies) at $16^{\circ} \mathrm{C}$ for $2 \mathrm{~h}$. Labeled RNA samples were dried in a vacuum concentrator for $1 \mathrm{~h}$. Once samples are completely dried, they were prepared for hybridization by reconstitution in a nuclease-free aqueous solution containing the appropriate concentrations of Hyb Spike-In solution, 10X GE Blocking Agent and 2X Hi-RPM Hybridization Buffer (Agilent Technologies). Reconstituted RNA samples ( $45 \mu 1 /$ sample) were added to microarrays and hybridization was achieved by rotation at $20 \mathrm{rpm}$ for $20 \mathrm{~h}$ at $55^{\circ} \mathrm{C}$. Subsequently, microarrays were washed using Agilent Gene Expression Wash Buffers 1 as recommended by the manufacturer, and scanned on an Agilent Technologies G4900DA SureScan scanner at 3- $\mu \mathrm{m}$ resolution.

Raw data preparation and statistical analysis. Raw microarray data were acquired and analyzed using Agilent Feature Extraction software (version 11.0.1.1). Using the software, raw data were summarized automatically to provide expression data for each gene probed on the microarray. Array data were filtered using a setting of gIsGeneDetected $=1$ for all samples. Selected miRNA gTotalGeneSignal walues were logarithmically transformed and normalized to allow comparison between arrays by using a quantile normalization method. Comparative analyses between test samples and control samples were performed by measuring fold-changes in relative gene expression levels. Hierarchical cluster analysis was performed using complete linkage and Euclidean distance as a measure of similarity. All data analysis and visualization of differentially expressed genes was conducted using R Statistical language software version. 2.15.0.

Next, we compared miRNA expression ratios of corresponding brain metastatic adenocarcinomas to the primary gastric adenocarcinomas to identify miRNAs that were upregulated or downregulated in all 8 cases of brain metastasis. Dysregulated miRNAs were analyzed using the online database search program, miRDB (http://www.mirdb.org/ mirdb) to identify their respective top-ranked target miRNAs.

Immunohistochemical (IHC) staining. IHC staining was performed with representative tissue sections using a Ventana BenchMark XT autostainer (Ventana Medical Systems, Inc., Tucson, AZ, USA) according to the manufacturer's suggested protocol. The following antibodies were used for IHC staining: a polyclonal anti-rabbit anti-human ZEB1 antibody (HPA 027524, 1/200 dilution; Sigma-Aldrich, St. Louis, MO, USA), a mouse monoclonal anti-human ZFHX1B (ZEB2) antibody (Clone Mo4, 1/100 dilution; Abgent, San Diego, CA, USA) and a mouse monoclonal anti-human E-cadherin antibody (Clone 36/E-cadherin, 1/50 dilution; BD Biosciences, San Jose, CA, USA). Two pathologists (S.H. Kim and J. Choi) reviewed the immunohistochemical findings without prior knowledge of the patient histories. The pathologists scored the observation of prominent ZEB1 or ZEB2 staining as positive expression of either protein. In addition, tissue sections were scored as positive for E-cadherin if prominent membranous E-cadherin expression was observed.

Generation of a mouse brain metastatic gastric adenocarcinoma model with in vivo selection of gastric adenocarcinoma cell lines

Cancer cell lines. Gastric cancer cell lines (MKN28, MKN74, SNU-16, SNU-638 and NCI-N87) were purchased from the 


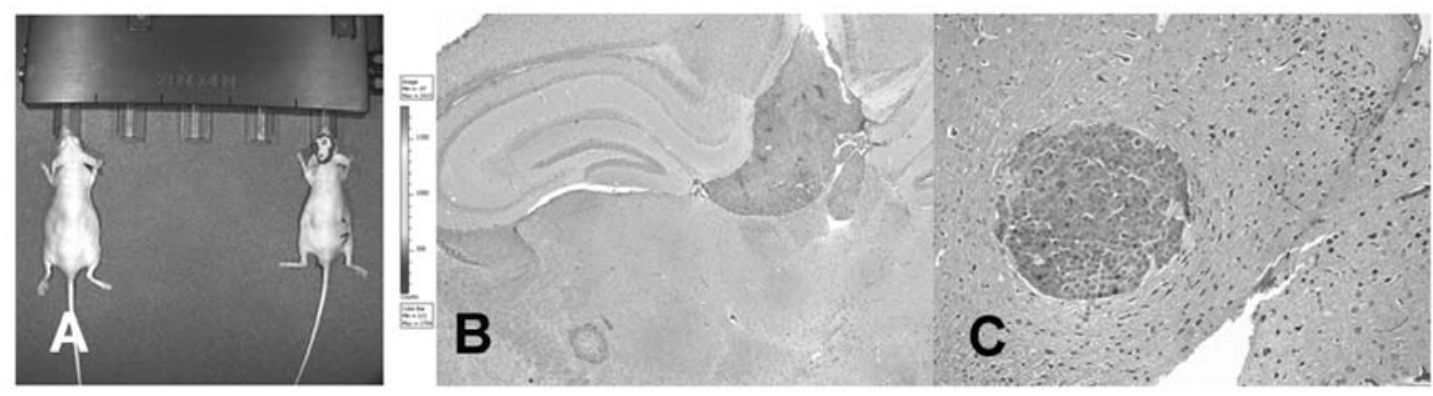

Figure 1. In vivo selection of brain metastatic gastric adenocarcinoma cell line (MKN74). (A) Gastric cancer cell lines stably expressing a luciferase reporter gene were introduced into 5-week-old BALB/c-nu mice by intracardiac injection. Two weeks following the injection, mice were anesthetized and injected intraperitoneally $(150 \mathrm{mg} / \mathrm{kg})$ with D-Luciferin solution. Shown are representative luciferase activities in the brain of a mouse injected with gastric tumor cells (right) and a control mouse without injection (left), as measured with an in vivo imaging system (IVIS). Metastatic carcinoma was identified in mice injected with gastric cancer cell lines (MKN74) (B, x40 magnification; C, x200 magnification). Metastatic cancer cells were isolated from mouse brain showing the highest IVIS signaling for subsequent use in selection of brain metastatic gastric adenocarcinoma cell lines in vivo.

Korean Cell Line Bank (Seoul, Korea). These cell lines were maintained in RPMI-1640 medium supplemented with $10 \%$ heat-inactivated fetal bovine serum and $1 \%$ penicillin/streptomycin.

Luciferase vector transfection. Gastric cancer cell lines were transfected with the pGL4.17 (luc2/Neo) vector (Promega, Madison, WI, USA) using Lipofectamine 2000 (Invitrogen, Carlsbad, CA, USA). Stable transfectants were generated by selection in G418 (Invitrogen) at $800 \mu \mathrm{g} / \mathrm{ml}$.

Mouse brain metastatic gastric adenocarcinoma model. Specific pathogen-free male BALB/c-nu mice (5 weeks old) were purchased from Central Lab. Animal, Inc. (Seoul, Korea) and quarantined for 1 week before entry into the study. Animal experiments were approved by the Institutional Animal Experiment Ethics Committee (2012-0276), and the care and use of mice were performed according to our institutional guidelines. Experimental brain metastases were established by intracardiac injection of $150 \mu \mathrm{l}$ of saline containing $2 \times 10^{6}$ tumor cells (MKN28, MKN74, SNU-16, SNU638 or NCI-N87 cells harboring the stably transfected pGL4.17 plasmid).

In vivo imaging system (IVIS). At two weeks post-injection with tumor cells, brain metastases were monitored weekly by the in vivo imaging system (IVIS; Xenogen IVIS 100; Caliper Life Sciences, Hopkinton, MA, USA). Briefly, mice were anesthetized with isoflurane gas and injected intraperitoneally $(150 \mathrm{mg} / \mathrm{kg}$ ) with D-Luciferin solution (VivoGlo Luciferin, \#P1041; Promega) and bioluminescent images were measured using an IVIS Spectrum (Fig. 1). Bioluminescent signals were quantified from ROIs using the Living Image software (Xenogen).

Isolation of cancer cells isolation from mouse brain. Animals were humanely sacrificed when brain metastases were prominently identified by IVIS monitoring. Mouse brains were gently harvested and metastatic tumor cells were obtained using the Cancer Cell Isolation kit (Panomics, Fremont, CA, USA). Briefly, mouse brains were carefully dissected using forceps and a scalpel. Metastatic tumor tissues were resuspended in tumor cell digestion solution and incubated at $37^{\circ} \mathrm{C}$ for $2-4 \mathrm{~h}$ with agitation. Single tumor cell suspensions were passed through a cell strainer and washed in tumor cell purification solution. Tumor cells were cultured in RPMI-1640 supplemented with $10 \%$ heat-inactivated fetal bovine serum and $1 \%$ penicillin/streptomycin and selected in $800 \mu \mathrm{g} / \mathrm{ml}$ G418, as done with the gastric cancer cells which were stably transfected with pGL4.17 vector. After 3-4 passages, metastatic brain tumor cells were injected into mice following the procedure described above for the purpose of in vivo selection. By serial injection of brain metastatic tumor cells, we derived second generation of brain metastatic tumor cells from the NCI-N87 cell line and a third generation of brain metastatic tumor cells from the MKN74 cell line.

Western blot analysis. Cells were lysed in cell extraction buffer [10 mM Tris, pH 7.4, $100 \mathrm{mM} \mathrm{NaCl}, 1 \mathrm{mM}$ EDTA, $1 \mathrm{mM}$ EGTA, $1 \mathrm{mM} \mathrm{NaF}, 20 \mathrm{mM} \mathrm{Na}_{4} \mathrm{P}_{2} \mathrm{O}_{7}, 2 \mathrm{mM} \mathrm{Na}_{3} \mathrm{VO}_{4}$, $1 \%$ Triton $\mathrm{X}-100,10 \%$ glycerol, $0.1 \%$ sodium dodecyl sulfate (SDS), $0.5 \%$ deoxycholate] in preparation for western blot analysis. Lysates containing $30 \mu \mathrm{g}$ of total protein were mixed with Laemmli sample buffer (Bio-Rad Laboratories, Hercules, CA, USA) and heated at $100^{\circ} \mathrm{C}$ for $5 \mathrm{~min}$. Samples were then resolved on 7\% SDS-polyacrylamide gel electrophoresis gels and electroblotted onto nitrocellulose membranes (Amersham Protran 0.45 NC; GE Healthcare Bio-Sciences, Pittsburgh, PA, USA). Membranes were blocked in 5\% non-fat dry milk in Tris-buffered saline with Tween-20 (Santa Cruz Biotechnology, Dallas, TX, USA), and incubated overnight at $4^{\circ} \mathrm{C}$ with a mouse monoclonal anti-human ZFHX1B antibody (Clone Mo4, 1/2,000 dilution; Abgent), a mouse monoclonal anti-human E-cadherin antibody (Clone 36/E-cadherin, $1 / 2,000$ dilution; BD Biosciences) or an anti- $\beta$-actin antibody (Clone AC-15, 1/50,000; Sigma-Aldrich). Subsequently, the nitrocellulose membranes were probed with peroxidaseconjugated goat anti-mouse IgG (sc-2055, 1/2,000; Santa Cruz Biotechnology) for $1 \mathrm{~h}$ at room temperature. Membranes were then washed and developed with a chemiluminescent agent (Amersham ECL Select; GE Healthcare Bio-Sciences).

\section{Results}

Patients. Sample blocks from 8 cases, of brain metastatic gastric adenocarcinoma ( 5 males and 3 females) were obtained from archival tissues stored at 3 hospitals. The age of patients 
Table I. Simultaneously increased or decreased miRNA expression ratio in matched brain metastatic gastric adenocarcinoma/ primary gastric adenocarcinoma.

\begin{tabular}{lccrrrrrr}
\hline & Case 1 & Case 2 & Case 3 & Case 4 & Case 5 & Case 6 & Case 7 & Case 8 \\
\hline $\begin{array}{l}\text { Overexpressed miRNAs } \\
\text { hsa-miR-106b-5p }\end{array}$ & 1.93 & 1.94 & 2.77 & 4.98 & 2.25 & 8.82 & 3.09 & 3.58 \\
hsa-miR-1260a & 2.72 & 2.22 & 14.98 & 7.08 & 6.49 & 15.61 & 1.67 & 3.12 \\
hsa-miR-141-3p & 1.35 & 2.17 & 52.85 & 3.49 & 3.11 & 9.43 & 2.19 & 1.79 \\
hsa-miR-19a-3p & 2.53 & 2.80 & 4.56 & 3.37 & 5.29 & 17.76 & 5.33 & 2.29 \\
hsa-miR-200b-3p & 4.27 & 2.31 & 52.19 & 2.30 & 2.61 & 4.01 & 1.74 & 3.49 \\
hsa-miR-93-5p & 2.90 & 1.76 & 3.57 & 6.08 & 2.90 & 10.22 & 4.05 & 5.19 \\
Underexpressed miRNAs & & & & & & & & \\
hsa-miR-4430 & -1.38 & -1.25 & -2.01 & -1.29 & -1.68 & -2.42 & -3.77 & -2.63 \\
hsa-miR-4689 & -1.26 & -1.89 & -8.55 & -2.92 & -2.87 & -8.55 & -1.05 & -1.03 \\
\hline
\end{tabular}

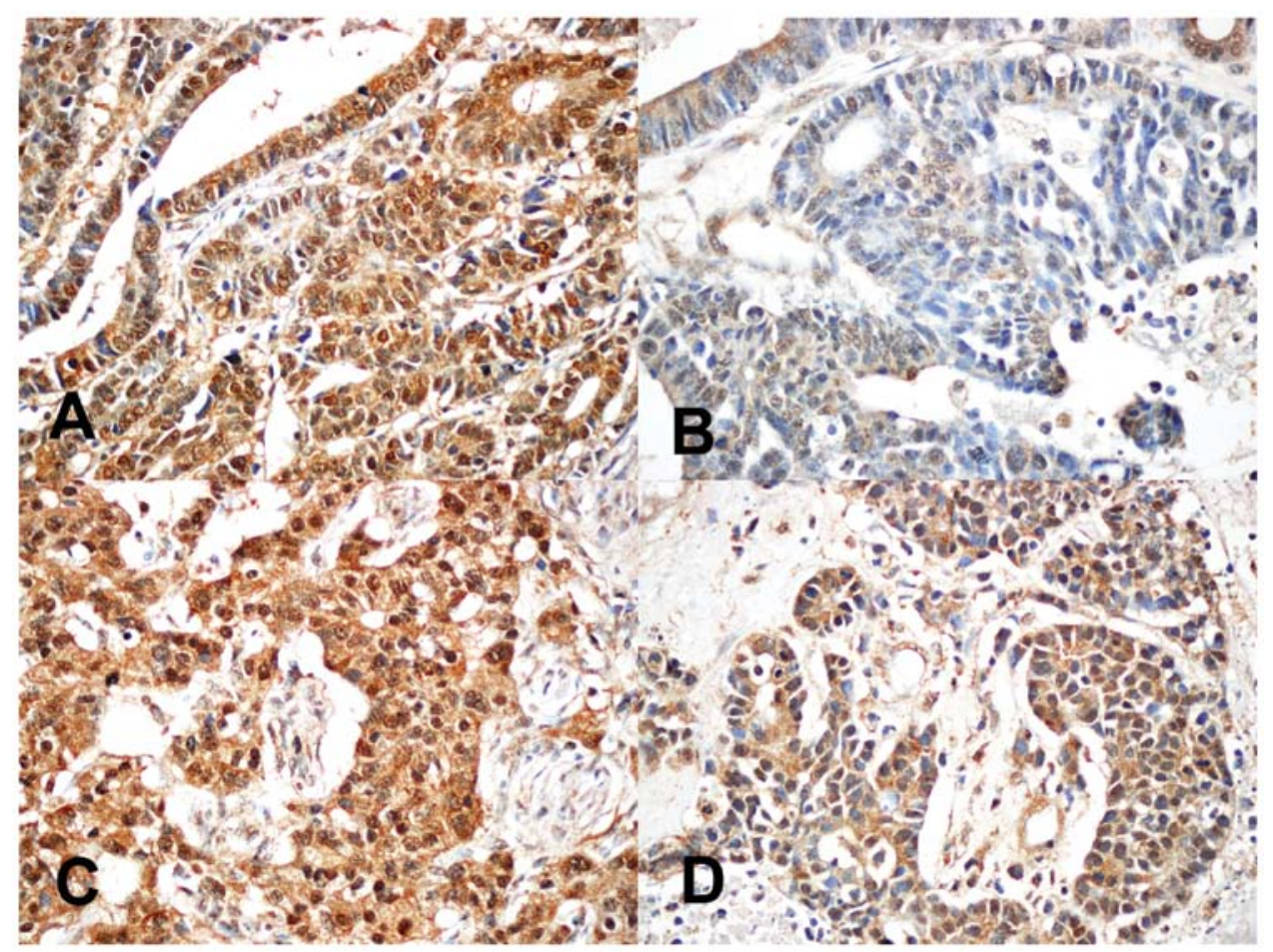

Figure 2. Loss of ZEB2 expression in brain metastatic gastric adenocarcinoma. Primary gastric adenocarcinoma (A, case 1, x400 magnification; C, case 2, $\mathrm{x} 400$ magnification) showed prominent nuclear ZEB2 immunopositivity. However, nuclear ZEB2 immunopositivity was reduced in brain metastases (B, case 1, x400 magnification; D, case 2, x400 magnification).

at the time of diagnosis with brain metastases ranged from 42 to 59 years, and the average survival period following diagnosis was 7 months. None of these cases showed HER2 overexpression, as determined by chromosomal in situ hybridization. Patients were classified as stage III (6 patients) and stage IV (2 patients). All 8 patients had deceased by the time the present study was initiated.

Analysis of microarray data. Microarray data analysis revealed 6 miRNAs that were upregulated and 2 were downregulated in the brain metastases samples (Table I). Next, we retrieved target gene information for the dysregulated miRNAs using the online database search program, miRDB (http://mirdb.
org/miRDB). The target genes for each miRNA are shown in Table II. The miRDB program revealed that $Z E B 2$ is the top-ranked target for hsa-miR-141-3p and hsa-miR-200b-3p, which are members of the miR-200 family (18). Therefore, we hypothesized that $Z E B 2$ might play an important role for brain metastases of gastric adenocarcinoma.

ZEB2 expression in primary gastric adenocarcinoma and corresponding brain metastases. To identify differences in ZEB2 expression between matched samples, we performed immunohistochemical staining for ZEB2 in 8 cases of gastric adenocarcinoma and the corresponding brain metastases. Among 8 cases of primary gastric adenocarcinoma, 4 cases 


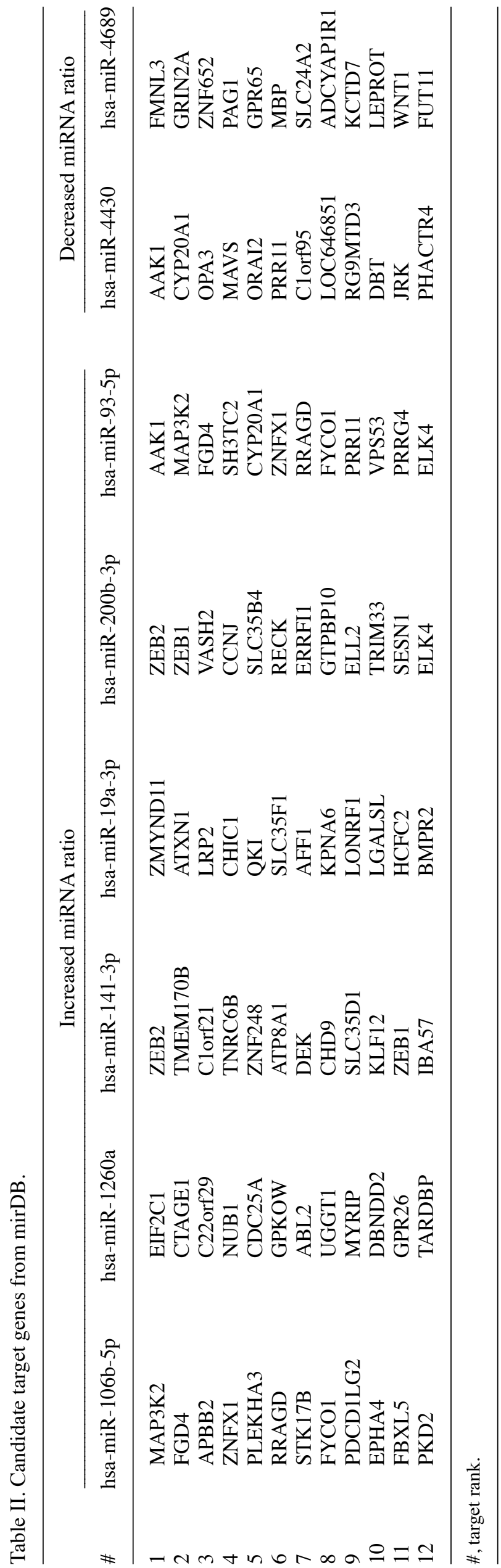

showed prominent nuclear ZEB2 expression. Notably, 3 of 4 cases showed markedly reduced nuclear ZEB2 expression in the corresponding brain metastatic gastric adenocarcinoma (Fig. 2). In one case, nuclear ZEB2 expression did not differ discernably between the primary and metastatic lesions (Table III). With respect to ZEB1, only 1 case of primary gastric adenocarcinoma showed nuclear expression. On the other hand, immunohistochemical staining results showed inconsistent results for expression of E-cadherin, which is repressed by ZEB1/ZEB2 $(19,20)$. In brain metastases, 2 cases showed increased E-cadherin expression, while the other 2 cases showed decreased E-cadherin expression (Fig. 3). The remaining 4 cases showed no differences in E-cadherin expression between primary and brain metastatic gastric adenocarcinomas. No consistent relationship between relative levels of ZEB2 and E-cadherin expression was observed in the metastatic brain lesions. Neither histologic differentiation, nor Lauren classification demonstrated a correlation between ZEB2 and E-cadherin expression (Table III).

Comparison of ZEB2 expression levels in parental and metastatic brain cell lines generated by in vivo selection. To confirm the differences observed in ZEB2 expression between primary and brain metastatic gastric adenocarcinoma, we generated 2 metastatic adenocarcinoma cell lines in mice by in vivo selection. Western blot analyses were performed to compare the two parental gastric adenocarcinoma cell lines (MKN74 and NCI-N87) with the in vivo-selected brain metastatic adenocarcinoma cell lines. We confirmed that ZEB2 expression in the 2nd and 3rd generation of MKN74 cells and 2nd generation of NCI-N87 cells was reduced, compared to the parental cell lines (Fig. 4). In the case of E-cadherin, MKN74 cell lines showed decreased expression with successive generations. In contrast, the NCI-N87 cell line did not express E-cadherin.

\section{Discussion}

Brain metastases in gastric adenocarcinoma have not been widely studies, primarily because of the relatively low incidence of brain metastases compared to lung and breast (21) and the difficulty in obtaining tumor samples, as most cases show leptomeningeal carcinomatosis, rather than mass-forming lesions (5-7). The low incidence of brain metastases likely results from the common treatment failure patterns following gastrectomy, characterized by locoregional recurrence, peritoneal carcinomatosis and liver metastasis (7), as opposed to distant metastases. Extra-abdominal metastasis is relatively rare. Considering these facts, one of the most important findings of the present study is the demonstration of feasibility of comparing matched primary gastric adenocarcinoma and brain metastatic lesions in patients.

To overcome the limitations of paraffin samples, we examined the differences in miRNA expression levels in brain metastatic lesions and, primary gastric adenocarcinoma. Fresh frozen tissues are the gold standard for miRNA analysis. However, it has been proposed that miRNA is an eminently suitable, i.e. stable, target molecule for analysis in FFPE tissues (22). Recently, several reports have discussed miRNA profiles in gastric adenocarcinoma samples including FFPE 
Table III. Histological characteristics and ZEB1, ZEB2 and E-cadherin immunohistochemical staining results.

\begin{tabular}{|c|c|c|c|c|c|c|c|c|}
\hline & \multirow[b]{2}{*}{ Differentiation } & \multirow[b]{2}{*}{ Lauren classification } & \multicolumn{2}{|c|}{ ZEB1 } & \multicolumn{2}{|c|}{ ZEB2 } & \multicolumn{2}{|c|}{ E-cadherin } \\
\hline & & & Stomach & Brain & Stomach & Brain & Stomach & Brain \\
\hline Case 1 & Moderate & Intestinal & - & - & + & - & - & + \\
\hline Case 2 & Poor & Mixed & - & - & + & - & + & + \\
\hline Case 3 & Poor & Mixed & + & - & - & - & + & + \\
\hline Case 4 & Moderate & Intestinal & - & - & - & - & + & + \\
\hline Case 5 & Moderate & Intestinal & - & - & - & - & - & + \\
\hline Case 6 & Poor & Diffuse & - & - & + & - & + & - \\
\hline Case 7 & Moderate & Intestinal & - & - & + & + & + & - \\
\hline Case 8 & Moderate & Intestinal & - & - & - & - & + & + \\
\hline
\end{tabular}

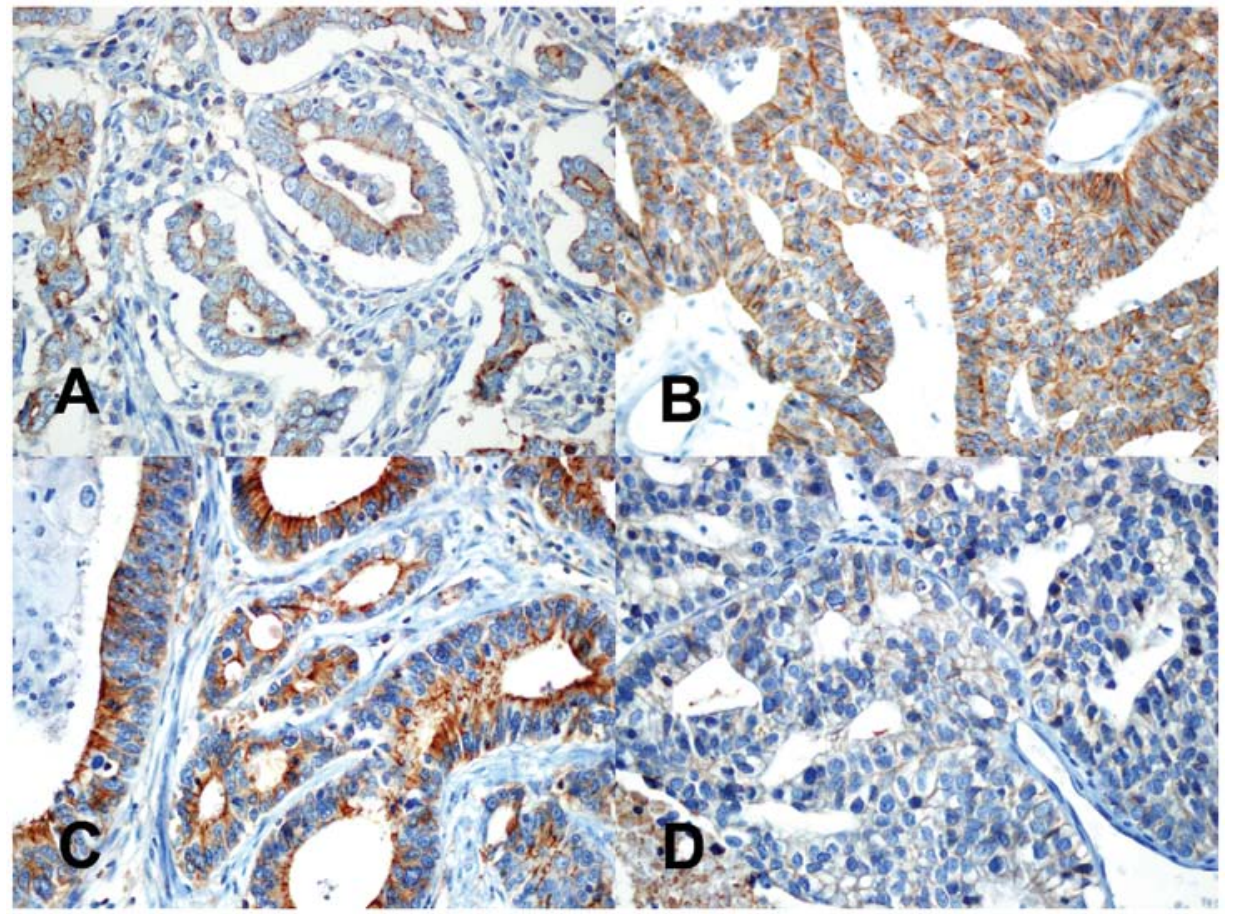

Figure 3. E-cadherin expression in brain metastatic gastric adenocarcinoma. Case 5 (A and B, x400 magnification) showed increased E-cadherin membranous immunopositivity after brain metastasis (B). In contrast, case 7 (C and D, 400x magnification) showed decreased E-cadherin immunopositivity after brain metastasis (D)

specimens $(17,20,23)$. However, studies designed to investigate the roles of miRNA in gastric adenocarcinoma metastasis have been rare (24), highlighting an additional significant element of this study.

In comparing miRNA profiles between primary gastric adenocarcinomas and corresponding brain metastatic carcinomas, we identified 6 upregulated miRNAs (hsa-miR-106b-5p, hsa-miR-1260a, hsa-miR-141-3p, hsamiR-19a-3p, hsa-miR-200b-3p and hsa-miR-93-5p) and 2 downregulated miRNAs (miR-4430 and miR-4389) in the brain metastatic samples. Among these miRNAs, hsamiR-141-3p and hsa-miR-200b-3p belong to the miR-200 family (18). The miR-200 family serves a critical role in the repression of E-cadherin by ZEB1/2 during EMT, thereby enhancing migration and invasion during cancer progression $(18,19,25,26)$. It is well known that EMT and metastases are associated with E-cadherin and ZEB1/2 in gastric adenocarcinoma $(20,27,28)$. Therefore, the upregulation of hsa-miR-141-3p and hsa-miR-200b-3p in the brain metastatic lesions may promote metastasis via mesenchymal-epithelial transition (MET) during the colonization of brain tissues. Online analysis programs allowed the identification of putative miRNA targets, revealing ZEB1/2 as candidate target molecules for brain metastases of gastric adenocarcinoma. To verify differences in ZEB1/2 expression, we performed immunohistochemical staining for each of the matched cases. ZEB1 was expressed in only 1 case of primary gastric adenocarcinoma without expression in the metastatic lesion. However, ZEB2 was expressed in 4 cases of primary gastric adenocarcinomas. Moreover, 3 of 4 corresponding brain metastatic adenocarcinoma samples showed loss of nuclear ZEB2 expression. ZEB2, also known as Smad-interacting 


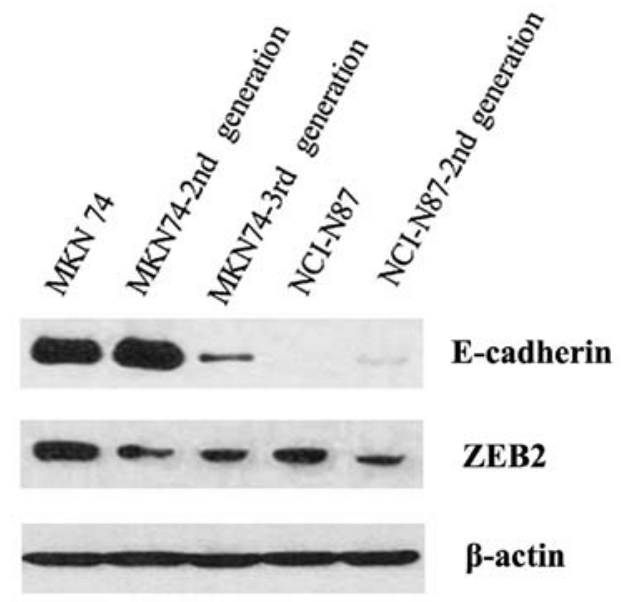

Figure 4. Western blot analysis of ZEB2 and E-cadherin expression with in vivo-selected brain metastatic gastric adenocarcinoma cell lines. The brain metastatic gastric adenocarcinoma cell lines (MKN74 and NCI-N87) showed decreased ZEB2 protein expression, compared to the respective parental cel lines. However, they did not show increased E-cadherin expression, compared to the respective parental cell lines.

protein 1 (SIP1), belongs to the zinc finger E-box binding protein (ZEB) family. ZEB2 plays an important role in EMT during embryonic development, as indicated by phenotypes observed in ZEB2 knockout mice (29). ZEB2 has been well demonstrated to bind the E-cadherin promoter and suppress the expression of this cell-cell adhesion molecule (30). Upregulation of ZEB2 mRNA is well demonstrated in various cancers. $Z E B 2 \mathrm{mRNA}$ levels are generally correlated with tumor metastasis, differentiation grade and poor prognosis (28). From these results, we propose that ZEB2 may play an important role in the development of brain metastases of gastric adenocarcinoma via MET.

In contrast with the ZEB2 IHC staining results, E-cadherin which is known to be regulated by ZEB2 (26), did not show any correlation in IHC staining. In addition, no discernable negative correlation was observed between E-cadherin and ZEB2 expression in western blots. Based on these results, we suggest that other, unknown mechanisms of regulating E-cadherin expression exist in brain metastatic gastric adenocarcinomas.

To confirm the observed differences of ZEB2 protein expression, we generated 2 brain metastatic gastric adenocarcinoma cell lines in mice by in vivo selection. By western blot analysis, we found that ZEB2 expression was decreased in brain metastatic adenocarcinoma cells, compared to their parental cell lines. Currently, there is no animal model for studying brain metastatic gastric cancer $(31,32)$. Although we did not prepare a stable, spontaneous metastatic cell line, we were able to generate 2 'transient' brain metastatic gastric cancer cell lines by intracardiac injection.

There are some potential limitations to the present study. The main limitation is that we could not study mRNA or protein expression levels in clinical patient samples because the samples were FFPE tissues. To overcome the limitation, we performed IHC staining with patient samples and western blot analysis with 2 cell lines simulating brain metastases in a clinical situation. The second potential limitation is that we observed lower ZEB1 expression in gastric adenocarcinoma cells by IHC than has been previously observed $(27,33)$. This difference may be due to the use of a different ZEB1 antibody or differences in criteria for interpreting result. We used strict criteria for interpreting IHC results as positive. The third limitation is that not all cases showed ZEB2 downregulation in the brain metastatic lesions. The mechanisms of brain metastases are very complex; thus, it is difficult to describe brain metastatic processes with a single hypothesis. The last limitation is that the inoculation method is important in studying EMT in experimental metastasis models. The injecting of tumor cells into the bloodstream of animals is unlikely to reflect changes in epithelial and mesenchymal gene expression levels seen in primary tumors prior to the metastatic process (32).

We conclude that the miRNA-200 family and ZEB2 may play a significant role in the development of brain metastases in gastric adenocarcinoma.

\section{Acknowledgements}

The present study was supported by a faculty research grants from the Yonsei University College of Medicine for 2013 (6-2013-0027) and the Basic Science Research Program through the National Research Foundation of Korea, funded by the Ministry of Education (2010-0021092) for Dr Se Hoon Kim. The authors appreciate Mr. Junyoung Park (Department of Nuclear Medicine) for his excellent technical support.

\section{References}

1. Boyle P and Levin B; International Agency for Research on Cancer and World Health Organization: World Cancer Report 2008. International Agency for Research on Cancer. WHO Press, Lyon/Geneva, 2008.

2. Bosman FT; World Health Organization and International Agency for Research on Cancer: WHO Classification of Tumours of the Digestive System. 4th edition. Bosman FT, Carneiro F, Hruban RH and Theise ND (eds). International Agency for Research on Cancer, Lyon, 2010.

3. Go PH, Klaassen Z, Meadows MC and Chamberlain RS: Gastrointestinal cancer and brain metastasis: a rare and ominous sign. Cancer 117: 3630-3640, 2011.

4. Zhang S, Wang M, Xue YH and Chen YP: Cerebral metastasis from hepatoid adenocarcinoma of the stomach. World $\mathrm{J}$ Gastroenterol 13: 5787-5793, 2007.

5. Oh SY, Lee SJ, Lee J, et al: Gastric leptomeningeal carcinomatosis: multi-center retrospective analysis of 54 cases. World $\mathrm{J}$ Gastroenterol 15: 5086-5090, 2009.

6. Tomita H, Yasui H, Boku N, et al: Leptomeningeal carcinomatosis associated with gastric cancer. Int J Clin Oncol 17: 361-366, 2011.

7. Lee JL, Kang YK, Kim TW, et al: Leptomeningeal carcinomatosis in gastric cancer. J Neurooncol 66: 167-174, 2004.

8. Gazzeri R, Galarza M, Neroni M and Gaaaeri G: Central Nervous System Metastases From Gastric Cancer. In: Research Focus on Gastric Cancer. Cardinni DC (ed). Nova Science Publishers, Inc., Hauppauge, NY, pp165-183, 2007.

9. Bartolotti M, Franceschi E and Brandes AA: EGF receptor tyrosine kinase inhibitors in the treatment of brain metastases from non-small-cell lung cancer. Expert Rev Anticancer Ther 12: 1429-1435, 2012.

10. Patel RR and Mehta MP: Targeted therapy for brain metastases: improving the therapeutic ratio. Clin Cancer Res 13: 1675-1683, 2007.

11. Weil RJ: Does trastuzumab increase the risk of isolated central nervous system metastases in patients with breast cancer? Nat Clin Pract Oncol 3: 236-237, 2006. 
12. Bang YJ, Van Cutsem E, Feyereislova A, et al: Trastuzumab in combination with chemotherapy versus chemotherapy alone for treatment of HER2-positive advanced gastric or gastro-oesophageal junction cancer (ToGA): a phase 3, open-label, randomised controlled trial. Lancet 376: 687-697, 2010.

13. Ohtsu A, Shah MA, Van Cutsem E, et al: Bevacizumab in combination with chemotherapy as first-line therapy in advanced gastric cancer: a randomized, double-blind, placebo-controlled phase III study. J Clin Oncol 29: 3968-3976, 2011.

14. Calin GA and Croce CM: MicroRNA signatures in human cancers. Nat Rev Cancer 6: 857-866, 2006.

15. Nicoloso MS, Spizzo R, Shimizu M, Rossi S and Calin GA: MicroRNAs - the micro steering wheel of tumour metastases. Nat Rev Cancer 9: 293-302, 2009.

16. Osawa S, Shimada Y, Sekine S, et al: MicroRNA profiling of gastric cancer patients from formalin-fixed paraffin-embedded samples. Oncol Lett 2: 613-619, 2011.

17. Liu A and Xu X: MicroRNA isolation from formalin-fixed, paraffin-embedded tissues. Methods Mol Biol 724: 259-267, 2011.

18. Korpal M,Lee ES,Hu G and Kang Y: The miR-200 family inhibits epithelial-mesenchymal transition and cancer cell migration by direct targeting of E-cadherin transcriptional repressors $Z E B$ and ZEB2. J Biol Chem 283: 14910-14914, 2008.

19. Song JH and Meltzer SJ: MicroRNAs in pathogenesis, diagnosis, and treatment of gastroesophageal cancers. Gastroenterology 143: 35-47.e32, 2012.

20. Cong N, Du P, Zhang A, et al: Downregulated microRNA-200a promotes EMT and tumor growth through the wnt $/ \beta$-catenin pathway by targeting the E-cadherin repressors ZEB1/ZEB2 in gastric adenocarcinoma. Oncol Rep 29: 1579-1587, 2013.

21. Nathoo N, Chahlavi A, Barnett GH and Toms SA: Pathobiology of brain metastases. J Clin Pathol 58: 237-242, 2005.

22. Klopfleisch R, Weiss AT and Gruber AD: Excavation of a buried treasure - DNA, mRNA, miRNA and protein analysis in formalin fixed, paraffin embedded tissues. Histol Histopathol 26: 797-810, 2011.
23. Shen R, Pan S, Qi S, Lin X and Cheng S: Epigenetic repression of microRNA-129-2 leads to overexpression of SOX4 in gastric cancer. Biochem Biophys Res Commun 394: 1047-1052, 2010.

24. Zhang Z, Liu S, Shi R and Zhao G: miR-27 promotes human gastric cancer cell metastasis by inducing epithelial-to-mesenchymal transition. Cancer Genet 204: 486-491, 2011.

25. Gregory PA, Bert AG, Paterson EL, et al: The miR-200 family and miR-205 regulate epithelial to mesenchymal transition by targeting ZEB1 and SIP1. Nat Cell Biol 10: 593-601, 2008.

26. Park SM, Gaur AB, Lengyel E and Peter ME: The miR-200 family determines the epithelial phenotype of cancer cells by targeting the E-cadherin repressors ZEB1 and ZEB2. Genes Dev 22: 894-907, 2008.

27. Jia B, Liu H, Kong Q and Li B: Overexpression of ZEB1 associated with metastasis and invasion in patients with gastric carcinoma. Mol Cell Biochem 366: 223-229, 2012.

28. Dai YH, Tang YP, Zhu HY, et al: ZEB2 promotes the metastasis of gastric cancer and modulates epithelial mesenchymal transition of gastric cancer cells. Dig Dis Sci 57: 1253-1260, 2012.

29. Van de Putte T, Maruhashi M, Francis A, et al: Mice lacking $Z F H X 1 B$, the gene that codes for Smad-interacting protein-1, reveal a role for multiple neural crest cell defects in the etiology of Hirschsprung disease-mental retardation syndrome. Am J Hum Genet 72: 465-470, 2003.

30. Comijn J, Berx G, Vermassen P, et al: The two-handed E box binding zinc finger protein SIP1 downregulates E-cadherin and induces invasion. Mol Cell 7: 1267-1278, 2001.

31. Cruz-Munoz W and Kerbel RS: Preclinical approaches to study the biology and treatment of brain metastases. Semin Cancer Biol 21: 123-130, 2011.

32. Daphu I, Sundstrom T, Horn S, et al: In vivo animal models for studying brain metastasis: value and limitations. Clin Exp Metastasis 30: 695-710, 2013

33. Ryu HS, Park do J, Kim HH, Kim WH and Lee HS: Combination of epithelial-mesenchymal transition and cancer stem cell-like phenotypes has independent prognostic value in gastric cancer. Hum Pathol 43: 520-528, 2012. 